\title{
COMPETÊNCIA AVALIATIVA DO ENFERMEIRO PARA O RECONHECIMENTO E ENFRENTAMENTO DAS NECESSIDADES EM SAÚDE DAS FAMÍLIAS*
}

\author{
Maria Marta Nolasco Chaves ${ }^{1}$, Emiko Yoshikawa Egry²
}

\begin{abstract}
RESUMO: Estudo exploratório, descritivo, ancorado na Teoria da Intervenção Práxica da Enfermagem em Saúde Coletiva, objetivou identificar a competência avaliativa do enfermeiro para o reconhecimento e o enfrentamento das necessidades em saúde das famílias. O local do estudo foi um Distrito Sanitário da capital paranaense. Os dados foram coletados em fontes primárias e secundárias no período de junho de 2008 a julho de 2009. Os participantes da pesquisa foram 25 enfermeiras de Unidades de Saúde. Os resultados indicam que o enfermeiro reconhece processos determinantes das necessidades em saúde e do processo saúde-doença nas diferentes dimensões da realidade, singular, particular e estrutural, porém ao intervir não os relaciona, e assim, direciona suas ações para a dimensão singular. Nas considerações finais os desafios apontados são no sentido de capacitar os profissionais para promover intervenções que busquem reverter os processos determinantes das necessidades em saúde dos usuários dos serviços de saúde local.

DESCRITORES: Competência profissional; Enfermeiros; Saúde pública; Atenção primária à saúde.
\end{abstract}

\section{THE NURSE'S EVALUATIVE COMPETENCE FOR RECOGNIZING AND FACING THEFAMILIES' HEALTH NEEDS}

\begin{abstract}
This exploratory descriptive study, anchored in the Theory of Praxis Intervention of Nursing in Collective Health, aimed to identify the nurse's evaluative competence for recognizing and facing the families' health needs. The study took place in a Health District in the capital of the state of Paraná, the data was collected from primary and secondary sources in the period June 2008 - July 2009, and the participants in the research were 25 nurses from Health Centers. The results indicate that the nurse recognizes determinant processes of the health needs and of the health-illness process in the different dimensions of the context - singular, particular, and structural, but that in intervening she does not relate them, and thus directs her actions to the singular dimension. In the final considerations, the challenges indicated are in the sense of training the professionals to promote interventions which seek to turn back the determinant processes of the health needs of the users of the local health services.

DESCRIPTORS: Professional competence; Nurses; Public health; Primary health care.

\section{COMPETENCIA EVALUATIVA DEL ENFERMERO PARA EL RECONOCIMIENTO Y AFRONTAMIENTO DE LAS NECESIDADES EN SALUD DE LAS FAMILIAS}

RESUMEN: Estudio exploratorio, descriptivo, fundamentado en la Teoría de la Intervención Práctica de la Enfermería en Salud Colectiva. Su objetivo fue identificar la competencia evaluativa del enfermero para el reconocimiento y afrontamiento de las necesidades en salud de las familias. El sitio del estudio fue un Distrito Sanitario de la capital de Paraná. Los datos fueron obtenidos en fuentes primarias y secundarias en el período de junio de 2008 a julio de 2009 y los participantes de la investigación fueron 25 enfermeras de Unidades de Salud. Los resultados apuntan que el enfermero reconoce procesos determinantes de las necesidades en salud y del proceso salud-enfermedad en las diferentes dimensiones de la realidad, singular, particular y estructural, pero al intervenir no los relaciona, y así, direcciona sus acciones para la dimensión singular. En las consideraciones finales los desafíos apuntados se relacionan al sentido de capacitar los profesionales para promover intervenciones que busquen reverter los procesos determinantes de las necesidades en salud de los usuarios de los servicios de salud local. DESCRIPTORES: Competencia profesional; Enfermeros; Salud pública; Atención primaria a la salud.

*Extraído da tese intitulada 'Competência avaliativa do enfermeiro para o reconhecimento e enfrentamento das necessidades em saúde das famílias', apresentada à Escola de Enfermagem de Ribeirão Preto da Universidade de São Paulo em 2010.

${ }^{1}$ Enfermeira. Doutora em Enfermagem. Professora do Departamento de Enfermagem e Programa de Pós-Graduação da Universidade Federal do Paraná.

${ }^{2}$ Enfermeira. Doutora em Enfermagem. Professora do Departamento de Enfermagem em Saúde Coletiva da Escola de Enfermagem da USP. Bolsista Produtividade CNPq.

Autor correspondente:

Recebido: $14 / 02 / 2013$

Maria Marta Nolasco Chaves

Aprovado: 10/11/2013

Universidade Federal do Paraná

Rua Pref. Lothário Meissner, 632 - 80210-170 - Curitiba-PR-Brasil

E-mail:mnolasco@terra.com.br 


\section{INTRODUÇÃO}

O processo saúde-doença é expresso diferentemente para cada classe social, pois este é o processo de desgaste biológico do sujeito pela sua inserção no processo de produção e nas relações de reprodução social ${ }^{(1)}$. As necessidades consideradas existenciais, naturais ou necessárias são aquelas referentes à manutenção da vida dos indivíduos em seu contexto histórico-social ${ }^{(2)}$.

A redução da compreensão de necessidades como algo expresso individual e biologicamente tem sido a prática do sistema institucionalizado de saúde. Esse é um processo que impede a reflexão sobre a determinação do processo saúde-doença e que autoriza ao Estado normatizar para intervir em saúde, definindo as demandas a serem atendidas nos serviços do setor, assim como, as necessidades em saúde a serem satisfeitas ${ }^{(3)}$.

Em uma sociedade capitalista as necessidades mesmo sendo necessárias para a vida só serão atendidas de acordo com as possibilidades e obstáculos que se têm em um espaço que é histórico e socialmente construído. Nestes espaços estarão as marcas dos condicionantes econômicos, culturais, políticos em cada classe social, caracterizado pelas relações de gênero e étnicas ${ }^{(1)}$.

Nas sociedades modernas foi difundida a noção de risco e esta passou a ser representada por cálculos, percepções e comunicação sobre o risco no sentido de prever um futuro controlável pela mudança de comportamento do indivíduo. Então, a informação e o monitoramento para vigiar e controlar os riscos estabeleceu nova forma de viver. Na saúde tais probabilidades foram direcionadas para os cálculos que representam características individuais que levam ao desenvolvimento de um evento adverso - a doença. Assim, determinados comportamentos, hábitos, estilos de vida devem ser abandonados pelos indivíduos. A análise de risco tem justificado a alocação de verbas públicas para atender às demandas de grupos com intervenções para diminuir a desigualdade social sem discutir políticas públicas mais amplas de inclusão social que possam ir contra o interesse de classes dominantes ${ }^{(4)}$.

A Teoria da Intervenção Práxica da Enfermagem em Saúde Coletiva (TIPESC), fundamenta a sistematização da assistência nas etapas que são captar, interpretar e intervir em um fenômeno observado na realidade objetiva. Para a captação e a interpretação desse fenômeno se referenda o reconhecimento de sua determinação pela compreensão da sua autoarticulação com o processo de produção e reprodução social. Neste sentido, considera que a intervenção em saúde modifica a realidade referindo que esta deverá ser novamente interpretada, e assim, dar continuidade ao processo interpretativo e de intervenção ${ }^{(5)}$.

Baseado nesses pressupostos que se reflete sobre a intervenção em saúde e a formação do profissional para desenvolvê-la, a qual deve ser contínua baseada em referenciais e saberes que permitam a autonomia do trabalhador e não a sua adaptação às condições socioeconômicas impostas. No atual modelo de competência para a formação do trabalhador referenda-se que a aprendizagem ocorre por meio da experiência no trabalho, com a valorização de competências individuais para atender ao modelo liberal. E neste modelo se perde a importância dos elementos da participação, da formação da consciência de classe e da possibilidade de transformar determinadas realidades ${ }^{(6-7)}$.

Portanto, investigar a competência avaliativa do enfermeiro para reconhecer e enfrentar as necessidades em saúde das famílias se justifica para contribuir para a formação deste profissional, como também permite refletir sobre as condições do trabalho que interferem nesse processo.

\section{MÉTODO}

Estudo exploratório descritivo com abordagem qualitativa, ancorado na TIPESC ${ }^{(5)}$. O universo da pesquisa foi em um Distrito Sanitário do município de Curitiba-PR. No estudo os aspectos éticos foram respeitados seguindo as normatizações para as pesquisas com seres humanos - resolução 196/96 do CNS. As aprovações foram obtidas nos processos 740/2008 Comitê de Ética em Pesquisa da Escola de Enfermagem da Universidade de São Paulo e 09/2008 do Comitê de Ética da Secretaria Municipal de Saúde de Curitiba.

A Coleta de dados empíricos foi por meio de entrevistas semiestruturadas coletadas no período de junho de 2008 a julho de 2009 . Os participantes foram 25 enfermeiras de 14 Unidades de Saúde do referido território que aceitaram participar do estudo por meio da assinatura do Termo de Consentimento Livre e Esclarecido.

A coleta de dados secundários foi baseada na proposta investigativa sobre a determinação do processo saúde doença ${ }^{(8)}$, com a qual se propôs reconhecer os processos de desgastes e de proteção presentes na vida da população moradora do Distrito. Além disso, foram analisados os discursos da participação popular por meio de dos relatórios das conferências municipais de saúde ocorridas no período de 1997 a 2007, bem como, foram analisados os protocolos de saúde vigentes no 
município, a fim de compreender a definição das intervenções de saúde no município, as quais norteiam a prática profissional.

A análise dos documentos públicos sobre as condições de vida da população, o discurso das conferências e dos protocolos de saúde foi fundamentada na hermenêutica dialética para compreender a complexidade das relações estabelecidas destes e a realidade encontrada ${ }^{(9)}$.

As fontes de dados secundários foram os bancos de dados públicos dos seguintes órgãos: Instituto Paranaense de Desenvolvimento Econômico e Social (IPARDES); Instituto de Pesquisa e Planejamento Urbano de Curitiba (IPPUC); Secretaria Municipal de Saúde de Curitiba (SMS-CTBA); Secretaria Estadual de Saúde do Paraná (SESA); Dados do Distrito Sanitário; Relatórios Finais das Conferências Municipais de Saúde ${ }^{(10-15)}$.

\section{RESULTADOS}

O reconhecimento das necessidades em saúde das famílias para as entrevistadas esteve relacionado à aproximação às realidades de saúde dos usuários, principalmente no desenvolvimento de visitas domiciliárias e visitas às comunidades das áreas de abrangência da Unidade de Saúde. Nessas, as observações realizadas pelos entrevistados eram sobre as condições de higiene pessoal e do ambiente, sobre a dinâmica da família diante de um agravo ou frente a uma situação nova que o grupo estaria vivendo:

[...] na visita domiciliar [...] observar a higiene do usuário e de sua casa [...] reconhecendo o papel dos membros das famílias [...] como a família se insere na comunidade [...] relato de ocorrências na área trazido pelo agente comunitário [...].

Pela questão social da família [...].

O agente comunitário foi reconhecido como o profissional que realizava visitas domiciliárias e visitas nas comunidades, o enfermeiro como o profissional que cumpria rotina de atividades na própria Unidade de Saúde e, consequentemente, para este profissional, a realização de atividades externas foi descrita como difícil. Nesse sentido, nos relatos das atividades dos agentes as entrevistadas tinham a possibilidade de reconhecer as realidades dos usuários e de suas famílias.

No discurso verificou-se que o desenvolvimento de atividades programáticas potencializava o reconheci- mento das necessidades em saúde pelos entrevistados. A implantação da Estratégia Saúde da Família (ESF) foi referida como potencial para o reconhecimento das necessidades. Entre as quatorze unidades de saúde das entrevistadas apenas três tinham a ESF implantada e o restante era Unidade Básica de Saúde (UBS). Outra potencialidade dos programas implantados para reconhecer as necessidades em saúde, segundo as entrevistadas, estava na elaboração dos registros e relatórios sobre as ações programáticas que realizavam. A articulação com outros profissionais ou serviços locais também foram citados como potencialidades para o reconhecimento de necessidades:

[...] sistema de referência e contra referência [...] apoio de técnicos do Distrito Sanitário para o desenvolvimento de intervenções [...] técnicos de serviços credenciados para o desenvolvimento de ações em saúde mental [...] rede de serviços para encaminhamentos [...].

As demandas espontâneas para os atendimentos e intervenções realizadas na Unidade de Saúde possibilitavam o reconhecimento de necessidades em saúde, segundo as entrevistadas. Nas intervenções descritas para aqueles que buscaram o serviço sem agenda prévia, foi relatado que as ações e atividades de caráter curativo eram as mais procuradas e ainda, que a consulta médica era a atividade com maior demanda. Também foi relatado que a não adesão dos usuários às intervenções de caráter preventivo realizadas nos serviços de atenção básica. A falta de recursos financeiros do usuário para o transporte ou para pagar serviços de apoio diagnóstico ou especializados foram considerados como necessidades em saúde.

A ocupação de alguns usuários com o recolhimento de lixo reciclável foi descrita como situação de risco à saúde dos usuários, das suas famílias, e também da comunidade. Reflexões sobre por que esta atividade passou a ser a única possibilidade de renda de algumas famílias daquelas comunidades não foram expressas, mas houve o reconhecimento da atividade como: árdua, insalubre e de significativo desgaste para aqueles que a executavam. Somado a esta reflexão, as entrevistadas reconheceram que tais indivíduos não estavam preparados para o trabalho por falta de escolaridade e de qualificação.

A violência em algumas famílias foi reconhecida como consequência do envolvimento dos sujeitos com o narcotráfico local e, algumas mortes nas comunidades foram relacionadas ao envolvimento da pessoa com o tráfico de drogas. Nas Conferências de Saúde a violên- 
cia passou a ser abordada a partir da 6.a Conferência Municipal de Saúde ${ }^{(12)}$. No evento seguinte, em 2003 ${ }^{(13)}$, houve a aprovação de proposta para a criação das Redes de Proteção para diferentes segmentos populacionais (idoso, criança e adolescente e mulher), nas quais os serviços de saúde passariam a participar em conjunto com instituições de outros setores.

Os dados secundários sobre morbimortalidade por causas externas em Curitiba mostraram que os índices de violência têm aumentado e que morte por causas externas representou a terceira causa de óbito no ano de 2006. Com relação aos óbitos por causas externas no ano referido, o grupo mais atingido foi do sexo masculino na faixa etária de 15 a 29 anos. No período de 2003 a 2006 a mulher foi a maior vítima de violência, sendo identificada a violência sexual como o tipo mais frequente ${ }^{(17)}$.

Quanto aos agravos descritos como necessidades em saúde os mais citados foram relacionados aos transtornos psiquiátricos, principalmente a dependência química, seguido de relatos sobre as doenças crônicas (hipertensão e diabetes), doenças infectocontagiosas (HIV, tuberculose, escabiose e verminose), usuários idosos portadores de dependências por complicações de doenças crônicas, gestação de alto risco e neoplasias. Todos esses agravos foram encontrados nos discursos das seis Conferências Municipais de Saúde, assim como nos protocolos vigentes na Secretaria Municipal:

[...] portadores de transtornos mentais por uso de drogas [...] hipertensão no adulto jovem [...] diabetes [...] feridas em pés de portadores de diabetes [...] amputação de membro [...] doenças infectocontagiosas [...] portadores de HIV grávidas [...] não adesão ao tratamento de HIV/Aids [...].

Quanto ao desenvolvimento de intervenções para o enfrentamento das necessidades em saúde reconhecidas as entrevistadas referiram a importância de se conhecer a realidade do usuário e da família sem impor valores pessoais, e assim, intervir. Nas situações descritas o reconhecimento da realidade ocorreu sobre aquilo que podia ser observado imediatamente no cotidiano dos indivíduos.

A mobilização das lideranças da comunidade, família e usuários para desenvolver intervenções foi apontada no sentido de que ao buscar esta ajuda as entrevistadas procuravam garantir recursos, os quais o Estado não garantia soluções na medida em que o usuário demandava. A parceria com a família para promover a intervenção foi referida como um meio par auxiliar a equipe de saúde para que o usuário a reconhecesse e enfrentasse os seus problemas de saúde.

Nas propostas aprovadas nas Conferências estudadas apontou-se para a responsabilização dos indivíduos e das famílias para o desenvolvimento do autocuidado e adoção de estilo de vida mais saudável como medidas para a promoção da saúde e enfrentamento aos agravos. Assim como se apontou a cor responsabilização do profissional da equipe local de saúde sobre os indicadores de saúde que deveriam ser modificados:

Recursos comunitários [...]. Verba arrecadada no bazar de doações realizado na comunidade [...] auxílio da comunidade de maneira geral [...] recursos de políticos locais [...] recursos financeiros do usuário para aquisição de medicamentos [...] compreensão sobre como funciona o complexo sistema burocrático do setor saúde [...] a família.

O trabalho em equipe com o objetivo de promover a integração entre os profissionais e, destes com os usuários, foi compreendido como potencialidade para a intervenção resolutiva em saúde. As entrevistadas referiram que há necessidade de capacitação da equipe para o desenvolvimento de intervenções, as quais deveriam ir além dos sinais e sintomas expressos por usuários portadores de agravos, quando refletiram sobre as suas próprias limitações para intervir diferentemente do modelo curativista vigente, pois demonstraram compreender que as condições socioeconômicas determinavam as condições de vida, o adoecimento e a morte dos moradores das áreas de abrangência das unidades de saúde e que, ao desenvolver algumas intervenções tinham limites para transformar aquelas realidades:

Equipe multiprofissional local [...] apoio e relacionamento entre os técnicos na Unidade de Saúde [...] reunião de equipe para discutir a questão de saúde do usuário e as intervenções a serem desenvolvidas [...].

As atividades de educação à saúde foram descritas como possibilidade para intervenções de promoção da saúde e prevenção de doenças. A articulação com outros setores e serviços de saúde de outros níveis de complexidade para promoverem intervenção foi referida no enfrentamento de questões instaladas. Os recursos para as ações com outros setoress foram relacionados à programas sociais que garantiam alimentos, cuidados no domicílio, inserção no trabalho e renda. As entrevistadas demonstraram que iam além daquilo que estava previsto nas normatizações dos serviços: 
[...] outras instituições: PROAMA, FAS, CRAS [...] programa do leite [...] rede de proteção à criança e a mulher [...] instituições de outros setores: creches, escolas, fundação cultural [...] cursos profissionalizantes [...] estagiários de universidades [...] voluntariado para ajudar em questões sociais [...].

Os recursos utilizados para desenvolver intervenções de enfrentamento às necessidades na Unidade de Saúde foram desde os conhecimentos do profissional mobilizados para intervir, a gestão local, até os materiais, equipamentos e estrutura física disponíveis para garantir a execução de intervenções. Os gestores da Secretaria Municipal e do Distrito Sanitário, assim como os programas de saúde, foram apontados como facilitadores para viabilizar desde insumos até os profissionais para compor a equipe local:

[...] conhecimentos pessoais para a orientação verbal [...] competências pessoais do enfermeiro para avaliar os casos [...]. Criatividade para usar os recursos segundo as possibilidades dos usuários [...].

Com relação às intervenções para a cura e prevenção de adoecimentos o destaque foi para os insumos previstos e disponibilizados pelos programas de saúde vigentes no município. Houve relato de dificuldades de recursos para o desenvolvimento da intervenção, no caso destaca-se essa dificuldade como um recurso, uma vez que, ao relatar a ausência ou precariedade de algum equipamento ou material necessário para a intervenção, a entrevistada descreveu outros recursos que buscou no sentido de realizar a ação.

\section{DISCUSSÃO}

Nos discursos a realidade foi compreendida sob dois polos. No primeiro polo, segundo a compreensão dos entrevistados, está o 'social' referente à realidade objetiva, em dados concretos no cotidiano dos indivíduos, tal como a inserção no processo de produção, as condições da moradia, o consumo de bens e produtos, as relações entre os membros da família e desta com a comunidade. No segundo polo está o "social" não concreto, não palpável, algo não específico e abrangente, distante daquele cotidiano, mas determinante da realidade objetiva reconhecida. Assim, no polo do concreto encontra-se a dimensão singular e, no polo do não concreto, as dimensões particular e estrutural da realidade, pois estas representaram os processos invisíveis no território.
Reconheceu-se que parte da realidade era percebida e descrita ao se elaborar os relatórios dos programas implantados, pois nesse momento o profissional refletia sobre os dados consolidados. Tal processo reforça a lógica da programação em saúde a qual tem organizado o quê fazer nos serviços de saúde para uma população-alvo específica e previamente estabelecida pelos critérios de inclusão no programa. Neste sentido, o profissional percebe aquilo que está previamente direcionado ou indicado para ser visto, e assim, é levado a desconsiderar a singularidades do processo saúde-doença dos indivíduos ${ }^{(1,4-5)}$.

Na caracterização de Curitiba e do Distrito Sanitário, observou-se as dimensões da realidade do território estava muito além daquilo que se podia observar no domicílio ${ }^{(18)}$. No espaço domiciliário não havia possibilidade de se encontrar concretamente as relações estabelecidas na produção que determinavam a reprodução social dos grupos e famílias, assim como, não seria possível ali reconhecer a historicidade dos processos de desgastes e fortalecimentos daqueles sujeitos. Portanto, inserção na produção e, consequentemente, o perfil de reprodução social não foram apontados como processo determinante das necessidades em saúde ${ }^{(1)}$. Apesar disso, houve referência à determinação social e econômica para as realidades descritas, tais como grupos homogêneos moradores de áreas consideradas de risco devido à falta de preparo e inserção no trabalho formal.

No território estudado, as comunidades das regiões mais periféricas dos bairros são as que vivem em terrenos de ocupação irregular e, assim, sem acesso regular à luz, saneamento básico e água tratada. Estas pequenas regiões também ficaram fora da política pública municipal chamada "linhão do emprego" que teve início no ano de 1997, a qual teve objetivo de apoiar empresas que se encontravam no mercado informal com infraestrutura nos polos empresariais ${ }^{(18)}$.

As intervenções desenvolvidas nas Unidades de Saúde para atender às necessidades reconhecidas estiveram relacionadas ao atendimento à demanda espontânea com predominância de atividades curativas e de apoio diagnóstico para os indivíduos com agravos já instalados. Para atender ao usuário se considerava a capacidade instalada na Unidade de Saúde e, principalmente, a possibilidade de agendar consulta médica, reforçando a centralidade da intervenção neste procedimento, fenômeno coerente com a própria lógica da programação em saúde que ancora a organização dos serviços.

Ao falarem de necessidades em saúde reconhecidas e enfrentadas junto às famílias as entrevistadas des- 
creveram as dinâmicas dos relacionamentos no núcleo familiar e a estrutura das famílias como processos protetores ou de desgastes para a saúde. Em muitas situações descritas não havia quem assumisse o papel de cuidador no domicílio, como também, em alguns casos não se tinha condições materiais mínimas para se desenvolver o cuidado. Porém, não se refletiu sobre o papel do Estado para prever tais recursos ${ }^{(1)}$.

$\mathrm{Na}$ análise dos protocolos e programas de saúde, foi observado que o avanço no discurso está relacionado à criação das Redes de Proteção para indivíduos em situação de risco para a violência com recomendação para intervenções multidisciplinares e intersetoriais ${ }^{(8-14)}$. Porém, o enfoque de risco as condições sociais e econômicas das famílias ficam apontadas apenas como fatores favoráveis e desencadeantes da violência ${ }^{(4)}$.

No enfrentamento de um evento inesperado, para um desempenho com iniciativa e criatividade, o trabalhador depende da mobilização dos seus recursos internos para escolher o melhor modo de agir, mas, sobretudo, depende dos recursos coletivos disponibilizados pela empresa ou instituição. Os recursos internos são aqueles que o próprio indivíduo adquire e desenvolve ao longo de sua formação pessoal e profissional e os recursos coletivos vão desde os aperfeiçoamentos, as formações proporcionadas pela empresa, como também as relações e o apoio de colegas para o trabalho ${ }^{(7)}$.

$\mathrm{Na}$ busca de resolver os problemas o trabalhador avalia a situação, os recursos necessários e, com as possibilidades que dispõe, julga se será viável intervir ou não. Logo, julga continuamente se conseguirá atingir os resultados desejados no sentido de mudar a situação que havia encontrado inicialmente ${ }^{(7)}$. Nesse sentido, no setor saúde é preciso que os profissionais desenvolvam a compreensão crítico-reflexiva sobre os fenômenos observados na realidade objetiva para refletirem sobre os processos determinantes do processo saúde-doença dos indivíduos, famílias ou grupos, segundo as relações de gênero, classe social, étnicas e culturais presentes em um território em um determinado tempo histórico para que proponham intervenções comprometidas com um projeto de transformação da realidade ${ }^{(1,5,7)}$.

\section{CONSIDERAÇÕES FINAIS}

No discurso dos entrevistados foi identificado que o reconhecimento das necessidades em saúde tem ênfase nos aspectos biológicos do processo de adoecimento, apesar da percepção sobre processos existentes na dimensão particular e estrutural como determinante do agravo. O referencial teórico que o profissional mobilizou para o reconhecimento de necessidades em saúde foi baseado na epidemiologia do risco, ou seja, nas probabilidades de desenvolvimento de dano à saúde do usuário e da família pelas condições de vida observadas no domicílio e no território, coerente com os protocolos de saúde vigentes no município.

Foi possível verificar o comprometimento dos entrevistados com os indivíduos e as famílias do território de sua responsabilidade. Porém, ao analisar a compreensão dos sujeitos sob a realidade descrita, encontra-se que esta é constituída daquilo que é mais aparente, mais próximo do seu cotidiano no trabalho. Ao descrever a intervenção desenvolvida os entrevistados demonstraram que privilegiam as ações de efeito mais imediato para aquela realidade, o que demonstra a dificuldade do profissional para promover intervenções que transformem a realidade.

Portanto, para que se tenha o desenvolvimento da competência avaliativa do enfermeiro em saúde coletiva é preciso proporcionar formações e capacitações fundamentadas em referenciais crítico-reflexivos que permitam ao profissional compreender os processos de proteção e de desgaste presentes nas diferentes dimensões da realidade, assim como nas diversas formas de viver.

\section{REFERÊNCIAS}

1. Breilh J. Epidemiologia crítica: ciência emancipadora e interculturalidade. Rio de Janeiro: Hucitec; 2006.

2. Heller A. Teoría da lasnecesidades em Marx. $2^{\mathrm{a}}$ ed. Barcelona: Península; 1986.

3. Stotz EM. Necessidades de saúde mediações de um conceito: contribuição das ciências para a fundamentação teórico-metodológica de conceitos operacionais da área de planejamento em saúde [tese]. Rio de Janeiro (RJ): Escola Nacional de Saúde Pública; 1991.

4. Luiz OC, Cohn A. Sociedade de risco e risco epidemiológico. Cad. Saúde Pública. 2006;22(11):2339-48.

5. Egry EY. Saúde coletiva: construindo um novo método em enfermagem. São Paulo: Ícone; 1996.

6. Ramos MN. A pedagogia das competências: autonomia ou adaptação. São Paulo: Cortez; 2002.

7. Zarifian P. O modelo da competência: trajetória histórica, desafios atuais e propostas. São Paulo: Senac; 2003.

Cogitare Enferm. 2013 Out/Dez; 18(4):729-35 
8. Breilh J, Tillería Y. Aceleración global y despojo enEcuador: El retroceso Del derecho a La vida y La salud pública enlas décadas neoliberales. QuitoEquador: Universidad Andina Simón Bolívar/AbyaAyla; 2009.

9. Minayo MCS. O desafio do conhecimento: pesquisa qualitativa em saúde. $8^{\text {a }}$ ed. São Paulo: Hucitec; 2004.

10. Prefeitura Municipal de Curitiba (PR). Secretaria Municipal de Saúde. Conselho Municipal de Saúde. Relatório da $4^{\text {a }}$ Conferência Municipal de Saúde Sistema Único de Saúde, saúde pública com qualidade. Curitiba; 1997.

11. Prefeitura Municipal de Curitiba (PR). Secretaria Municipal de Saúde. Conselho Municipal de Saúde. Relatório final da $5^{\text {a }}$ Conferência Municipal de Saúde SUS no III milênio em defesa da vida. Curitiba; 1999.

12. Prefeitura Municipal de Curitiba (PR). Secretaria Municipal de Saúde. Conselho Municipal de Saúde.

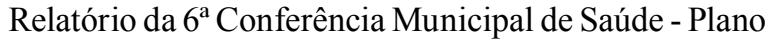
Municipal de Saúde de Curitiba 2002-2005. Curitiba; 2001.

13. Prefeitura Municipal de Curitiba (PR). Secretaria Municipal de Saúde. Conselho Municipal de Saúde. Relatório final da $7^{\text {a }}$ Conferência Municipal de Saúde - 15 anos do SUS Curitiba, conquistas, avanços e desafios. Curitiba; 2003.

14. Prefeitura Municipal de Curitiba (PR). Secretaria Municipal de Saúde. Conselho Municipal de Saúde. Relatório final da $8^{\text {a }}$ Conferência Municipal de Saúde - a participação popular na construção de um viver saudável. Curitiba; 2005.

15. Prefeitura Municipal de Curitiba (PR). Secretaria Municipal de Saúde. Conselho Municipal de Saúde. Relatório final da $9^{\text {a }}$ Conferência Municipal de Saúde - saúde e qualidade de vida: política de estado e desenvolvimento. Curitiba; 2008.

16. Fiorin JL. Linguagem e ideologia. $8^{\mathrm{a}}$ ed. São Paulo: Ática; 2005.

17. Secretaria Municipal de Saúde (PR). Boletim Epidemiológico de Curitiba. Curitiba; 2008b (ed. esp.).

18. Instituto de Pesquisa e Planejamento Urbano. Curitiba em dados. [acesso em 14 jul 2008]. Disponível: http:// ippucnet.ippuc.org.br/Bancodedados/ Curitiba em dados/Curitiba_em_dados_Pesquisa.asp. 